\title{
A Signal Transmission Model for Diffusion Based Molecular Communication Nanonetworks
}

\author{
Daniel Nzinga Kinsumuna, ${ }^{1, a^{*}}, \mathrm{D}$. Turgay Altilar ${ }^{1, \mathrm{~b}}$, Deniz Demiray ${ }^{1, \mathrm{c}}$. \\ ${ }^{1}$ Istanbul Technical University Informatics Institute \\ ITU Ayazaga Campus, 34469, Maslak, Istanbul, Turkey. \\ akinsumuna@yahoo.fr, baltilar@itu.edu.tr, 'cdemirayde@itu.edu.tr
}

\begin{abstract}
Keywords: Molecular Communication, Diffusion Based Molecular Communication, Nanonetworks, rational harvesting, confined environment.
\end{abstract}

\begin{abstract}
Nanonetworks is a communication between nanodevices, called nanomachines. Nanomachines are very small devices at the range of $10^{-9}$ metre. Molecular communication is one of the types of nanonetworks in which nanomachines are able to sense, calculate, actuate and intercommunicate. Transmission, propagation and reception are the three main stages to provide information exchange between sender and receiver in Molecular communication. Diffusion based molecular communication, that is an essential type of molecular communication, relies on propagation of molecules between sender and receiver nodes through free diffusion. This paper investigates how harvesting nodes can degrade the signal strength of the receiver node in a confined space. The analytic expression of the received signal at the receiver node has been drawn; the effects of the increasing number of harvesting nodes and the harvesting rate have been discussed in this paper.
\end{abstract}

\section{Introduction}

Molecular communication appeared to be an alternative to electromagnetic communication, enabling interaction among nanomachines [1]. However, research on molecular communication is not widespread. Molecular communication has been both inspired by biological communication systems and performed by biological cells. Therefore, biological cell based communication has to be investigated at biological cell level. In [2], an extensive survey on molecular nanonetworking paradigms has been introduced. In [3], the concept of molecular communication is introduced and the design of a molecular communication system has been sketched. In [4], diffusion-based molecular communications, which is the most widely used molecular communication technique, resources propagate by means of diffusion in a fluid medium, according to Fick's laws of diffusion [5], has been investigated. Distance effect of molecular harvesting over signal reception and harvesting performance in DIRECT, which results are based on one harvesting node, and also focuses on the effect of the harvester node location, is discussed in [6]. The main objective of this paper is two folds: first, the effect of multiple harvesters on signal strength at the receiving node is investigated. Furthermore, the harvesting rate over signal deterioration is studied. Secondly, a generalized analytic expression on the signal reception at the receiving node is proposed, which takes into account multiple harvesting nodes. Scientists in diffusion based molecular communication may use that analytic expression proposed in this current paper when dealing with nanonetworks composed of more than two receiving nodes. The rest of the paper is organized as follows. The model description is presented in section II. In section III, the analytic expression that computes the concentration of receiver node in a scenario of multiple harvesters is proposed. The results are discussed in section IV. Finally, a conclusion has been drawn based on the results obtained at section IV in section V. 


\section{Model description}

The bounded operating environment is a closed space, in which particles released by the transmitter node circulate in it such that when a particle reaches boundaries, it is bounced back in to the working environment. The total amount of particles within the environment does not change along the time. The transmitter node sends a signal, which is the input of the model, by releasing a number $(Q)$ particles. It is assumed that released particles are subject to only a random movement, called Brownian motion, in one dimensional bounded fluidic space. Therefore, the propagation of particles between transmitter and receiver nodes is uniquely accomplished by the Brownian motion. In order to analyse the impact of harvesters a discrete time system is defined. Within this model it is assumed that an harvester would keep only a percent of particles available in its range. The model is to investigate the impact of harvesters on signal reception at the receiving node. The receiver node measures the concentration within its location, which is subject to the signal strength in its range.

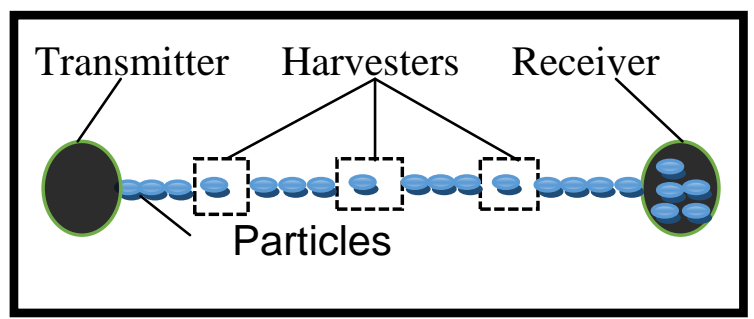

Fig. 1: Bounded space in 1D.

In this paper, the analysis for different number of harvester nodes has been done. Ultimately the impact of harvested particles on signal reception at the receiver node is analysed.

\section{Analysis}

In this paper the impact of increasing number of harvesting nodes and that of the harvesting rate over signal reception at the receiving node have been observed. The sender node emits a pulse consisting of $Q$ particles. The emitted pulse propagates over the working environment according to Eq. 1, with respect to Fick's laws of diffusion, which will eventually reach at the receiver node.

$$
c(x, t, Q)=\frac{Q}{(\sqrt{4 D \pi t})^{\sigma / 2}} e^{\frac{-x^{2}}{4 D t}} .
$$

where $Q$ is the amount of particles emitted by sender node, also called pulse amplitude, $x$ represents the distance between sender and receiver, $t$ stands for the time elapsed since emission of the signal, $c$ is the concentration of particles at location $x$ and time $t, D$ represents the diffusion coefficient of the working environment, $\sigma$ is the dimensional working space.

\section{III.1 Harvesters with finite capacity}

Assume that harvesting nodes are located between sender and receiver nodes, and that harvesters have a finite capacity of $\mathrm{H}$ particles. Although all received particles will be used later in the course of operation. The receiver would not have the chance of receiving the harvested particles. Since particles are kept in the harvester temporary the number of particles has been reduced to $R$. Therefore, the receiver would be under the diffusion of that many particles which yields to a new equation for diffusion (Eq. 2).

$$
c(x, t, Q)=\frac{R}{(\sqrt{4 D \pi t})^{\sigma / 2}} e^{-x^{2} / 4 D t} .
$$


where: $R=Q-\sum_{i=1}^{n} H_{i}\left(x_{i}, t\right)$.

$$
H_{i}\left(x_{i}, t\right)=\left\{\begin{array}{l}
0 \text { if } i=0 . \\
c\left(x_{i}, t, Q\right) \text { if } i=1 . \\
c\left(x_{i}, t, Q-\sum_{j=1}^{i-1} H_{j}\left(x_{j}, t\right)\right) \text { if } i>1 .
\end{array}\right.
$$

$n$ stands for the total number of harvesting nodes,

$i$ is an integer number from the range of 1 to $n$ and $x_{i}$ is the distance from the sender node to the harvesting node $i$ such that $x_{1}<x_{2}<x_{3} \ldots<x_{n-1}<x_{n}$.

$H_{i}$ represents the concentration held at harvesting node $i$ at position $x_{i}$ and time.

\section{III.2. Rational harvesting}

In the case that the harvesting nodes are placed at the same location with sender or receiver nodes, it is more likely that all particles from sender node will be absorbed by harvesters at instant time $t=0$. In this case, no resource will reach the receiving node. On the other hand, if harvesters are placed at the same location with the receiver node, unless harvesters' capacity be limited, otherwise, the receiving node will receive no particle. Thus, this is called irrational harvesting.

Harvesting nodes have limited capacity such that each harvester may only harvest up to $\beta \%$ of the received particles. This scenario is called rational harvesting. At each instant time $t$, each harvester cannot harvest more than $\beta \%$ of the received particles. Hence, Eq. 2 becomes

$$
c(x, t, Q)=\frac{R \frac{\beta}{100}}{(\sqrt{4 D \pi t})^{\sigma / 2}} e^{-x^{2} / 4 D t} .
$$

$\beta$ depends on the nature of the harvester and is named as harvesting parameter. If $\beta$ is too small, then there will be no significant harvesting impact on the signal reception at the receiving node The higher values for $\beta$ will yield impact on the signal strength at the receiving node.

\section{Simulations results}

MATLAB R2013a has been used as a tool to validate the proposed analytic expressions and visualize the impact of harvesting rate as well as number of harvesting nodes on the signal reception at the receiving node.

For the given parameters in Table 1 and values $\mathrm{A}$ and $\mathrm{B}$, the results have shown that the signal strength deteriorates, becomes worse in quality for $n=187$ (labeled as $187 \mathrm{HN}$ in Fig. 2). As shown in Fig. 2 for $n=3$, there is no much change in signal strength at the receiving node. It can be seen that as the number of harvesting nodes increases, the received signal decreases. This rule is valid for all locations where harvesting nodes may be placed at.

Table 1: Parameters and values used in simulations

\begin{tabular}{|c|c|c|}
\hline Parameters & Values A & Values B \\
\hline$Q$ & 500000 particles & 500000 particles \\
\hline$x$ & $1500 \mathrm{~nm}$ & $1500 \mathrm{~nm}$ \\
\hline$x_{i}$ & {$[10,600,1190]$} & {$[10,600,1190] ;[10: 30: 1480] ;[10: 8: 1498]$} \\
\hline$t$ & From 1 to $4 * 10^{5} \mathrm{~ns}$ & From 1 to $4 * 10^{5} \mathrm{~ns}$ \\
\hline$D$ & 1 & 1 \\
\hline$\sigma$ & 1 & $25 ; 50 ; 80$ \\
\hline$\beta$ & - & $3 ; 50 ; 187$ \\
\hline$n$ & 3 & \\
\hline
\end{tabular}



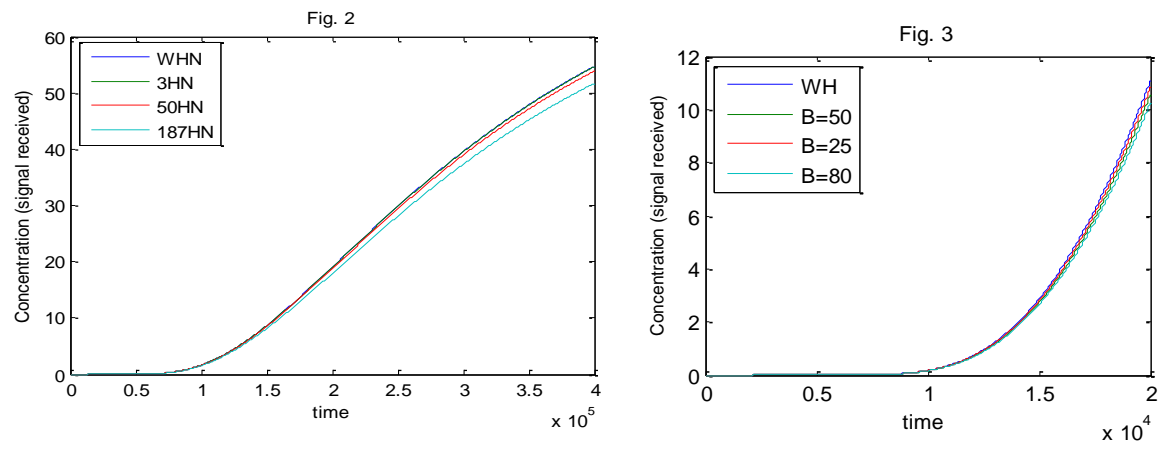

The $t$ axis is the time direction and the ordonate axis represents the received signal that is concentration actually. The legend indicates the signal strength for different number of Harvester Nodes $(\mathrm{HN})$ and harvesting rate $(\beta)$ in Fig. 2 and Fig. 3 respectively. Parameters' values in Fig. 3 shows that the harvesting rate is very important to preserve the signal strength at the receiving point. The red line is closer to the blue line, which is the expected signal (concentration) without harvesting. This is due to the fact that the lesser harvesting rate is used, the better signal strength is gotten.

\section{Conclusion}

The current study provides an analysis of diffusion based molecular communication nanonetwork which takes into account the effects of the number of harvesting nodes over signal reception at the receiving node. In addition, this paper has examined the harvesting rate at which harvesters must harvest particles without affecting the signal strength. The proposed generalised analytic expressions which compute the received signal for both rational and infinite capacity harvesting. Our research has found out that the received signal strength deteriorates progressively as the number of harvesting nodes increases. The results have also found that a significant number of particles may be harvested without much affecting the received signal strength at the receiving node if and only if the harvesting rate is as small as possible. Choosing a limited number of harvesting nodes and adopting for a small harvesting rate are the key for wiser harvesting without causing remarkable signal corruption at the receiving node. The future research will focus on the optimal number of harvesting nodes needed for wiser particles harvesting without deteriorating the signal strength.

\section{REFERENCES}

[1] I. F. Akyildiz, F. Fekri, R. Sivakumar, C. R. Forest, B. K. Hammer, Monaco: Fundamentals of Molecular Nano-communication Networks, IEEE Wireless Communications, 19(5):12-18, October 2012.

[2] I.F. Akyildiz, F. Brunetti, C. Bl'azquez, Nanonetworks: A new communication paradigm, Computer Networks, 52(12):2260-2279, August 2008

[3] M. Moore, A. Enomoto, T. Nakano, R. Egashira, T. Suda, A. Kayasuga, H. Kojima, H. Sakakibara, K. Oiwa, A Design of a Molecular Communication System for Nanomachines Using Molecular Motors, Fourth Annual IEEE International Conference on Pervasive Computing and Communications Workshops (PERCOMW'06), pp.:554-559, 2006.

[4] I. Llatser, A. Cabellos-Aparicio and E. Alarc'on, Networking Challenges and Principles in Diffusionbased Molecular Communication, IEEE Wireless Communications, 19(5):36-41, 2012.

[5] J. Philibert, One and a Half Century of Diffusion: Fick, Einstein, Before and Beyond, Diffusion Fundamentals 4 (2006) $6.1-6.19$

[6] D. Demiray, Distance Effect of Molecular Harvesting Over Signal Reception and Harvesting Performance in Direct, submitted for publication to Nano Communication Networks, 2014. 